\title{
Finding Small Roots of Bivariate Integer Polynomial Equations: A Direct Approach
}

\author{
Jean-Sébastien Coron \\ University of Luxembourg
}

\begin{abstract}
Coppersmith described at Eurocrypt 96 an algorithm for finding small roots of bivariate integer polynomial equations, based on lattice reduction. A simpler algorithm was later proposed in [9], but it was asymptotically less efficient than Coppersmith's algorithm. In this paper, we describe an analogous simplification but with the same asymptotic complexity as Coppersmith. We illustrate our new algorithm with the problem of factoring RSA moduli with high-order bits known; in practical experiments our method is several orders of magnitude faster than 9 .
\end{abstract}

Keywords: Coppersmith's theorem, lattice reduction, cryptanalysis.

\section{Introduction}

At Eurocrypt 96, Coppersmith described how lattice reduction can be used to find small roots of polynomial equations [5,6]. Coppersmith's technique has found numerous applications for breaking variants of RSA; for example, cryptanalysis of RSA with $d<N^{.29}$ [3], polynomial-time factorization of $N=p^{r} q$ for large $r$ [4, and cryptanalysis of RSA with small secret CRT-exponents [181]. Coppersmith's technique was also used to obtain an improved security proof for OAEP with small public exponent 23], and to show the deterministic equivalence between recovering the private exponent $d$ and factoring $N$ [10 19].

There are two main theorems from Coppersmith. The first one concerns finding small roots of $p(x)=0 \bmod N$ when the factorization of $N$ is unknown. Coppersmith proved that any root $x_{0}$ with $\left|x_{0}\right|<N^{1 / \delta}$ can be found in polynomial time, where $\delta=\operatorname{deg} p$. The technique consists in building a lattice that contains the solutions of the modular polynomial equation; all small solutions are shown to belong to an hyperplane of the lattice; an equation of this hyperplane is obtained by considering the last vector of an LLL-reduced basis; this gives a polynomial $h(x)$ such that $h\left(x_{0}\right)=0$ over the integers, from which one can recover $x_{0}$. The method can be extended to handle multivariate modular polynomial equations, but the extension is heuristic only.

Coppersmith's algorithm was further simplified by Howgrave-Graham in 13 . Howgrave-Graham's approach is more direct and consists in building a lattice of polynomials that are multiples of $p(x)$ and $N$; then by lattice reduction one computes a polynomial with small coefficients such that $h\left(x_{0}\right)=0 \bmod N^{k}$; if the coefficient of $h(x)$ are sufficiently small then $h\left(x_{0}\right)=0$ must hold over $\mathbb{Z}$ as 
well, which enables to recover $x_{0}$. Howgrave-Graham's approach seems easier to analyze, in particular for the heuristic extension to multivariate modular equations, for which there is much more freedom in selecting the polynomial multiples than for the univariate case. Howgrave-Graham's approach was actually used in all subsequent applications of Coppersmith's technique [13418 19 20].

Coppersmith's second theorem concerns finding small roots of bivariate integer polynomial equations $p(x, y)=0$ over the integers (not modulo $N$ ). Coppersmith proved that if $\left|x_{0}\right|<X$ and $\left|y_{0}\right|<Y$ with $X Y<W^{2 /(3 \delta)}$ then such root $\left(x_{0}, y_{0}\right)$ can be found in polynomial-time, where $W:=\max _{i j}\left|p_{i j}\right| X^{i} Y^{j}$. As for the univariate case, the algorithm consists in building a lattice containing the solutions of the polynomial equation; all small solutions are shown to belong to an hyperplane of the lattice, that is obtained by considering the last vector of an LLL-reduced basis. The equation of the hyperplane gives another polynomial $h(x, y)$ with the same root $\left(x_{0}, y_{0}\right)$ as $p(x, y)$, which enables to recover $\left(x_{0}, y_{0}\right)$. There can be improved bounds depending on the shape of the polynomial $p(x, y)$; see 2 for a complete analysis. As for the univariate case, the method extends heuristically to more variables. However, as mentioned in [8], the analysis is more difficult to follow than for the univariate case.

For Coppersmith's second theorem, a simplification was later proposed at Eurocrypt 2004 9], analogous to Howgrave-Graham's simplification for the univariate case. It consists in generating an arbitrary integer $n$ of appropriate size and constructing a lattice of polynomials that are multiples of $p(x, y)$ and $n$; then by lattice reduction one computes a polynomial with small coefficients such that $h\left(x_{0}, y_{0}\right)=0 \bmod n$; if the coefficients of $h(x, y)$ are sufficiently small, then $h\left(x_{0}, y_{0}\right)=0$ holds over $\mathbb{Z}$, which enables to recover $\left(x_{0}, y_{0}\right)$ by taking the resultant of $h(x, y)$ and $p(x, y)$. As for the univariate case, this approach seems easier to implement; it was later used in [11] for partial key exposure attacks on RSA, and in [16] to break one variant of RSA.

However, as opposed to the univariate case, this later simplification is not fully satisfactory because asymptotically its complexity is worse than for Coppersmith's second theorem. Namely, the algorithm in 9 is polynomial time under the stronger condition $X Y<W^{2 /(3 \delta)-\varepsilon}$, for any constant $\varepsilon>0$; but for $X Y<W^{2 /(3 \delta)}$ the algorithm has exponential-time complexity :

$$
\exp \left(\mathcal{O}\left(\log ^{2 / 3} W\right)\right)
$$

whereas Coppersmith's algorithm is polynomial time.

Therefore in this paper we describe a new algorithm for the bivariate integer case, with a simplification analogous to Howgrave-Graham and [9], but with the same polynomial-time complexity as in Coppersmith's algorithm; namely for $X Y<W^{2 /(3 \delta)}$ our algorithm has complexity

$$
\mathcal{O}\left(\log ^{15} W\right)
$$

using LLL 17] and $\mathcal{O}\left(\log ^{11} W\right)$ using the improved $L^{2}$ algorithm [21. This is done by taking a well chosen integer $n$ (rather than arbitrary) when building the 
lattice of polynomials; this enables to eliminate most columns of the lattice and then apply LLL on a sub-lattice of smaller dimension. Our new algorithm is easy to implement and performs well in practice. In Section 4 we show the results of practical experiments for the factoring with high-order bits known attack against RSA; we show that the running time is improved by several orders of magnitude compared to 9 .

\section{Preliminaries}

Let $u_{1}, \ldots, u_{\omega} \in \mathbb{Z}^{n}$ be linearly independent vectors with $\omega \leq n$. A lattice $L$ spanned by $\left\langle u_{1}, \ldots, u_{\omega}\right\rangle$ is the set of all integer linear combinations of $u_{1}, \ldots, u_{\omega}$. Such a set of vectors $u_{i}$ 's is called a lattice basis. We say that the lattice is full rank if $\omega=n$.

Any two bases of the same lattice $L$ are related by some integral matrix of determinant \pm 1 . Therefore, all the bases have the same Gramian determinant $\operatorname{det}_{1 \leq i, j \leq \omega}<u_{i}, u_{j}>$. One defines the determinant of the lattice $L$ as the square root of the Gramian determinant. If the lattice $L$ is full rank, then the determinant of $L$ is equal to the absolute value of the determinant of the $\omega \times \omega$ matrix whose rows are the basis vectors $u_{1}, \ldots, u_{\omega}$.

Theorem 1 (LLL). Let $L$ be a lattice spanned by $\left(u_{1}, \ldots, u_{\omega}\right) \in \mathbb{Z}^{n}$, where the Euclidean norm of each vector is bounded by B. The LLL algorithm, given $\left(u_{1}, \ldots, u_{\omega}\right)$, finds in time $\mathcal{O}\left(\omega^{5} n \log ^{3} B\right)$ a vector $b_{1}$ such that:

$$
\left\|b_{1}\right\| \leq 2^{(\omega-1) / 4} \operatorname{det}(L)^{1 / \omega} .
$$

In order to obtain a better complexity, one can use an improved version of LLL due to Nguyen and Stehlé, called the $L^{2}$ algorithm [21]. The $L^{2}$ algorithm achieves the same bound on $\left\|b_{1}\right\|$ but in time $\mathcal{O}\left(\omega^{4} n(\omega+\log B) \log B\right)$.

In this paper we also consider lattices generated by a set of vectors that are not necessarily linearly independent. Let $u_{1}, \ldots, u_{m} \in \mathbb{Z}^{n}$ with $m \geq n$; the lattice $L$ generated by $\left\langle u_{1}, \ldots, u_{m}\right\rangle$ consists of all integral linear combinations of $u_{1}, \ldots, u_{m}$. A lattice basis for $L$ can be obtained by triangularization of $u_{1}, \ldots, u_{m}$; a polynomial-time triangularization algorithm is described in [12]; more details will be given in Section 3.1

We prove a simple lemma that will be useful when analyzing the determinant of such lattices; it shows that the determinant of a full rank lattice generated by a matrix of row vectors is not modified when performing elementary operations on the columns of the matrix :

Lemma 1. Let $M$ be an integer matrix with $m$ rows and $n$ columns, with $m \geq n$. Let $L$ be the lattice generated by the rows of $M$. Let $M^{\prime}$ be a matrix obtained by elementary operations on the columns of $M$, and let $L^{\prime}$ be the lattice generated by the rows of $M^{\prime}$. Then if $L$ is full rank, $L^{\prime}$ is full rank with $\operatorname{det} L^{\prime}=\operatorname{det} L$.

Proof. See Appendix. 


\section{Our New Algorithm}

We consider a polynomial $p(x, y)$ with coefficients in $\mathbb{Z}$ with maximum degree $\delta$ independently in $x, y$ :

$$
p(x, y)=\sum_{0 \leq i, j \leq \delta} p_{i, j} x^{i} y^{j} .
$$

We are looking for an integer pair $\left(x_{0}, y_{0}\right)$ such that $p\left(x_{0}, y_{0}\right)=0$ and $\left|x_{0}\right|<X$ and $\left|y_{0}\right|<Y$. We assume that $p(x, y)$ is irreducible over the integers.

Let $k$ be an integer $>0$. We consider the set of polynomials :

$$
\begin{array}{ll}
s_{a, b}(x, y)=x^{a} \cdot y^{b} \cdot p(x, y), & \text { for } 0 \leq a, b<k \\
r_{i, j}(x, y)=x^{i} \cdot y^{j} \cdot n, & \text { for } 0 \leq i, j<k+\delta
\end{array}
$$

where the integer $n$ is generated in the following way.

Let indexes $\left(i_{0}, j_{0}\right)$ be such that $0 \leq i_{0}, j_{0} \leq \delta$; let $S$ be the matrix of row vectors obtained by taking the coefficients of the polynomials $s_{a, b}(x, y)$ for $0 \leq a, b<k$, but only in the monomials $x^{i_{0}+i} y^{j_{0}+j}$ for $0 \leq i, j<k$. There are $k^{2}$ such polynomials $s_{a, b}(x, y)$ and $k^{2}$ such monomials, so the matrix $S$ is a square matrix of dimension $k^{2}$ (see Figure 1 for an illustration); we take :

$$
n:=|\operatorname{det} S| \text {. }
$$

We will show in Lemma 3 that for a well chosen $\left(i_{0}, j_{0}\right)$, the value $|\operatorname{det} S|$ is lower bounded; in particular, this implies that $|\operatorname{det} S|>0$ and therefore matrix $S$ is invertible.

$S=$\begin{tabular}{c||cccc} 
& $x^{2} y^{2}$ & $x^{2} y$ & $x y^{2}$ & $x y$ \\
\hline \hline$s_{1,1}(x, y)$ & $a$ & $b$ & $c$ & $d$ \\
$s_{1,0}(x, y)$ & & $a$ & & $c$ \\
$s_{0,1}(x, y)$ & & & $a$ & $b$ \\
$s_{0,0}(x, y)$ & & & & $a$
\end{tabular}

Fig. 1. Matrix $S$ with $p(x, y)=a x y+b x+c y+d$, for $k=2$ and $\left(i_{0}, j_{0}\right)=(1,1)$. We get $n=|\operatorname{det} S|=a^{4}$.

Let $h(x, y)$ be a linear combination of the polynomials $s_{a, b}(x, y)$ and $r_{i, j}(x, y)$. Since we have that $s_{a, b}\left(x_{0}, y_{0}\right)=0 \bmod n$ for all $a, b$ and $r_{i, j}\left(x_{0}, y_{0}\right)=0 \bmod n$ for all $i, j$, we obtain :

$$
h\left(x_{0}, y_{0}\right)=0 \bmod n .
$$

The following lemma, due to Howgrave-Graham [13, shows that if the coefficients of polynomial $h(x, y)$ are sufficiently small, then $h\left(x_{0}, y_{0}\right)=0$ holds over the integers. For a polynomial $h(x, y)=\sum_{i, j} h_{i j} x^{i} y^{j}$, we define $\|h(x, y)\|^{2}:=$ $\sum_{i, j}\left|h_{i j}\right|^{2}$. 
Lemma 2 (Howgrave-Graham). Let $h(x, y) \in \mathbb{Z}[x, y]$ which is a sum of at most $\omega$ monomials. Suppose that $h\left(x_{0}, y_{0}\right)=0 \bmod n$ where $\left|x_{0}\right| \leq X$ and $\left|y_{0}\right| \leq Y$ and $\|h(x X, y Y)\|<n / \sqrt{\omega}$. Then $h\left(x_{0}, y_{0}\right)=0$ holds over the integers.

Proof. We have:

$$
\begin{aligned}
\left|h\left(x_{0}, y_{0}\right)\right| & =\left|\sum h_{i j} x_{0}^{i} y_{0}^{i}\right|=\left|\sum h_{i j} X^{i} Y^{j}\left(\frac{x_{0}}{X}\right)^{i}\left(\frac{y_{0}}{Y}\right)^{j}\right| \\
& \leq \sum\left|h_{i j} X^{i} Y^{j}\left(\frac{x_{0}}{X}\right)^{i}\left(\frac{y_{0}}{Y}\right)^{j}\right| \leq \sum\left|h_{i j} X^{i} Y^{j}\right| \\
& \leq \sqrt{\omega}\|h(x X, y Y)\|<n
\end{aligned}
$$

Since $h\left(x_{0}, y_{0}\right)=0 \bmod n$, this gives $h\left(x_{0}, y_{0}\right)=0$.

We consider the lattice $L$ generated by the row vectors formed with the coefficients of polynomials $s_{a, b}(x X, y Y)$ and $r_{i, j}(x X, y Y)$. In total, there are $k^{2}+$ $(k+\delta)^{2}$ such polynomials; moreover these polynomials are of maximum degree $\delta+k-1$ in $x, y$, so they contain at most $(\delta+k)^{2}$ coefficients. Let $M$ be the corresponding matrix of row vectors; $M$ is therefore a rectangular matrix with $k^{2}+(k+\delta)^{2}$ rows and $(k+\delta)^{2}$ columns (see Figure2 for an illustration). Observe that the rows of $M$ do not form a basis of $L$ (because there are more rows than

\begin{tabular}{|c|c|c|c|c|c|c|c|c|c|c|}
\hline & & $x^{2} y^{2}$ & $x^{2} y$ & $x y^{2}$ & $x y$ & $x^{2}$ & $y^{2}$ & $x$ & $y$ & 1 \\
\hline & $\begin{array}{l}s_{1,1}(x X, y Y) \\
s_{1,0}(x X, y Y) \\
s_{0,1}(x X, y Y) \\
s_{0,0}(x X, y Y)\end{array}$ & $\mid a X^{2} Y^{2}$ & $\begin{array}{l}b X^{2} Y \\
a X^{2} Y\end{array}$ & $\begin{array}{l}c X Y^{2} \\
a X Y^{2}\end{array}$ & $\begin{array}{l}d X Y \\
c X Y \\
b X Y \\
a X Y\end{array}$ & $b X^{2}$ & $c Y^{2}$ & $\begin{array}{l}d X \\
b X\end{array}$ & $\begin{array}{l}d Y \\
c Y\end{array}$ & $d$ \\
\hline$M=$ & $\begin{array}{l}r_{2,2}(x X, y Y) \\
r_{2,1}(x X, y Y) \\
r_{1,2}(x X, y Y) \\
r_{1,1}(x X, y Y) \\
r_{2,0}(x X, y Y) \\
r_{0,2}(x X, y Y) \\
r_{1,0}(x X, y Y) \\
r_{0,1}(x X, y Y) \\
r_{0,0}(x X, y Y)\end{array}$ & $n X^{2} Y^{2}$ & $n X^{2} Y$ & $n X Y^{2}$ & $n X Y$ & $n X^{2}$ & $n Y^{2}$ & $n X$ & $n Y$ & $n$ \\
\hline
\end{tabular}
columns), but $L$ is a full rank lattice of dimension $(k+\delta)^{2}$ (because the row vectors corresponding to polynomials $r_{i, j}(x X, y Y)$ form a full rank lattice).

Fig. 2. Lattice of polynomials with $p(x, y)=a x y+b x+c y+d$, for $k=2$ and $\left(i_{0}, j_{0}\right)=$ $(1,1)$

Let $L_{2}$ be the sublattice of $L$ where the coefficients corresponding to all monomials of the form $x^{i_{0}+i} y^{j_{0}+j}$ with $0 \leq i, j<k$ are set to zero (those monomials correspond to the matrix left-hand block in Fig. 2). There are $k^{2}$ such monomials, so $L_{2}$ is a full rank lattice of dimension : 


$$
\omega=(\delta+k)^{2}-k^{2}=\delta^{2}+2 \cdot k \cdot \delta .
$$

A matrix basis for $L_{2}$ can be obtained by first triangularizing $M$ using elementary row operations and then taking the corresponding submatrix (see Fig. 3). A polynomial-time triangularization algorithm is described in [12]; more details will be given in Section 3.1

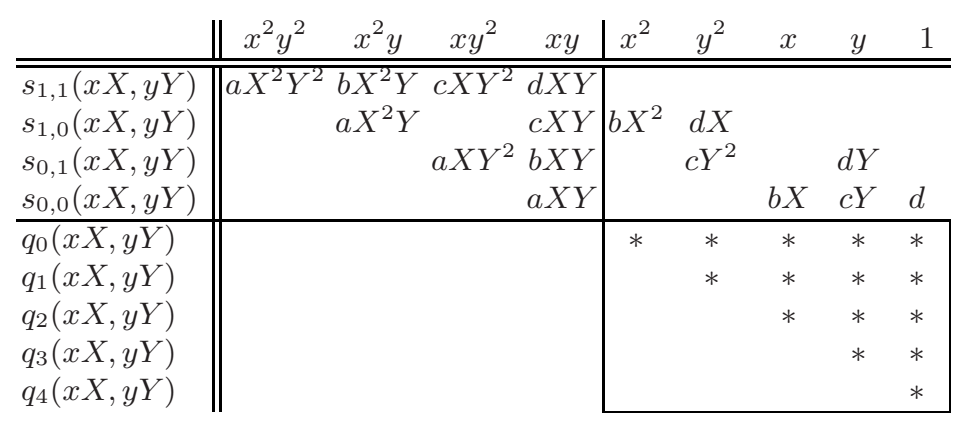

Fig. 3. Triangularized lattice of polynomials with $p(x, y)=a x y+b x+c y+d$, for $k=2$ and $\left(i_{0}, j_{0}\right)=(1,1)$. The 5 polynomials $q_{i}(x X, y Y)$ generate lattice $L_{2}$, with coefficients only in the 5 monomials $x^{2}, y^{2}, x, y$ and 1 . Algorithm LLL is applied on the corresponding 5 -dimensional lattice.

We apply the LLL algorithm on lattice $L_{2}$. From theorem 1, we obtain a non-zero polynomial $h(x, y)$ that satisfies $h\left(x_{0}, y_{0}\right)=0 \bmod n$ and :

$$
\|h(x X, y Y)\| \leq 2^{(\omega-1) / 4} \cdot \operatorname{det}\left(L_{2}\right)^{1 / \omega} .
$$

From lemma 2, this implies that if :

$$
2^{(\omega-1) / 4} \cdot \operatorname{det}\left(L_{2}\right)^{1 / \omega} \leq \frac{n}{\sqrt{\omega}}
$$

then $h\left(x_{0}, y_{0}\right)=0$ must hold over the integers.

Now we claim that polynomial $h(x, y)$ cannot be a multiple of $p(x, y)$. Assume the contrary; then the row vector coefficients of $h(x, y)$ is a linear combination of the row vector coefficients of polynomials $s_{a, b}(x, y)$ only. Given that matrix $S$ contains the coefficients of $s_{a, b}(x, y)$ for monomials $x^{i+i_{0}} y^{j+j_{0}}$ and given that $h(x, y)$ does not contain such monomials (because $h(x, y)$ lies in $L_{2}$ ), this gives a linear combination of the rows of $S$ equal to zero with non-zero coefficients; a contradiction since matrix $S$ is invertible.

The polynomial $p(x, y)$ being irreducible, this implies that $p(x, y)$ and $h(x, y)$ are algebraically independent with a common root $\left(x_{0}, y_{0}\right)$; therefore, taking :

$$
Q(x)=\operatorname{Resultant}_{y}(h(x, y), p(x, y))
$$

gives a non-zero integer polynomial such that $Q\left(x_{0}\right)=0$. Using any standard root-finding algorithm, we can recover $x_{0}$, and finally $y_{0}$ by solving $p\left(x_{0}, y\right)=0$. This terminates the description of our algorithm. 
It remains to compute the determinant of lattice $L_{2}$. First we consider the same matrices of row vectors as previously, except that we remove the $X^{i} Y^{j}$ powers. Therefore let $M^{\prime}$ be the same matrix as $M$, except that we take the coefficients of polynomials $s_{a, b}(x, y)$ and $r_{i, j}(x, y)$, instead of $s_{a, b}(x X, y Y)$ and $r_{i, j}(x X, y Y)$; matrix $M^{\prime}$ has $k^{2}+(k+\delta)^{2}$ rows and $(k+\delta)^{2}$ columns. We put the coefficients corresponding to monomials $x^{i+i_{0}} y^{j+j_{0}}$ for $0 \leq i, j<k$ on the left hand block, which has therefore $k^{2}$ columns; matrix $M^{\prime}$ has then the following form :

$$
M^{\prime}=\left[\begin{array}{cc}
S & T \\
n I_{k^{2}} & 0 \\
0 & n I_{w}
\end{array}\right]
$$

where $S$ is the previously defined square matrix of dimension $k^{2}$, while $T$ is a matrix with $k^{2}$ rows and $\omega=k^{2}+2 k \delta$ columns. Let $L^{\prime}$ be the lattice generated by the rows of $M^{\prime}$, and let $L_{2}^{\prime}$ be the sublattice where all coefficients corresponding to monomials $x^{i+i_{0}} y^{j+j_{0}}$ for $0 \leq i, j<k$ are set to zero. Note that lattice $L^{\prime}$ corresponds to lattice $L$ without the $X^{i} Y^{j}$ powers, whereas lattice $L_{2}^{\prime}$ corresponds to lattice $L_{2}$.

Since $n=|\operatorname{det} S|$, we can find an integer matrix $S^{\prime}$ satisfying :

$$
S^{\prime} \cdot S=n I_{k^{2}}
$$

namely $S^{\prime}$ is (up to sign) the adjoint matrix (or comatrix) of $S$, verifying $S^{\prime}$. $S=(\operatorname{det} S) I_{k^{2}}$. By elementary operations on the rows of $M^{\prime}$, we can therefore subtract $S^{\prime} \cdot S$ to the $n I_{k^{2}}$ block of $M^{\prime}$; this gives the following matrix :

$$
M_{2}^{\prime}=\left[\begin{array}{ccc}
I_{k^{2}} & 0 & 0 \\
-S^{\prime} & I_{k^{2}} & 0 \\
0 & 0 & I_{\omega}
\end{array}\right] \cdot M^{\prime}=\left[\begin{array}{cc}
S & T \\
0 & T^{\prime} \\
0 & n I_{\omega}
\end{array}\right]
$$

where $T^{\prime}=-S^{\prime} \cdot T$ is a matrix with $k^{2}$ rows and $\omega$ columns. By elementary operations on the rows of $M_{2}^{\prime}$, we obtain :

$$
M_{3}^{\prime}=U \cdot M_{2}^{\prime}=\left[\begin{array}{cc}
S & T \\
0 & T^{\prime \prime} \\
0
\end{array}\right]
$$

where $T^{\prime \prime}$ is a square matrix of dimension $\omega$. We obtain that $T^{\prime \prime}$ is a row matrix basis of lattice $L_{2}^{\prime}$, which gives :

$$
\operatorname{det} L^{\prime}=\left|\operatorname{det}\left[\begin{array}{cc}
S & T \\
0 & T^{\prime \prime}
\end{array}\right]\right|=|\operatorname{det} S| \cdot\left|\operatorname{det} T^{\prime \prime}\right|=|\operatorname{det} S| \cdot \operatorname{det} L_{2}^{\prime}=n \cdot \operatorname{det} L_{2}^{\prime} \text {. }
$$

We now proceed to compute $\operatorname{det} L^{\prime}$. The polynomial $p(x, y)$ being irreducible, the gcd of its coefficients is equal to 1 . This implies that by elementary operation of the columns of $M^{\prime}$, we can obtain a matrix whose left upper $k^{2} \times k^{2}$ block is the identity matrix and the right upper block is zero. From lemma 1, this does 
not change the determinant of the generated lattice. Let $V$ be the corresponding unimodular transformation matrix of dimension $(\delta+k)^{2}$; this gives :

$$
M_{4}^{\prime}=M^{\prime} \cdot V=\left[\begin{array}{cc}
I_{k^{2}} & 0 \\
n V
\end{array}\right] .
$$

By elementary row operations on $M_{4}^{\prime}$ based on $V^{-1}$ we obtain :

$$
M_{5}^{\prime}=\left[\begin{array}{cc}
I_{k^{2}} & 0 \\
0 & V^{-1}
\end{array}\right] \cdot M_{4}^{\prime}=\left[\begin{array}{lll}
I_{k^{2}} & 0 \\
& n I_{(\delta+k)^{2}}
\end{array}\right]=\left[\begin{array}{cc}
I_{k^{2}} & 0 \\
n I_{k^{2}} & 0 \\
0 & n I_{\omega}
\end{array}\right],
$$

which again by elementary row operations gives :

$$
M_{6}^{\prime}=U^{\prime} \cdot M_{5}^{\prime}=\left[\begin{array}{cc}
I_{k^{2}} & 0 \\
0 & n I_{\omega} \\
0 & 0
\end{array}\right]
$$

Finally this implies :

$$
\operatorname{det} L^{\prime}=\operatorname{det}\left[\begin{array}{cc}
I_{k^{2}} & 0 \\
0 & n I_{\omega}
\end{array}\right]=n^{\omega}
$$

Combining equations (77) and (8), we obtain :

$$
\operatorname{det} L_{2}^{\prime}=n^{\omega-1} \text {. }
$$

Recall that the columns of $L_{2}^{\prime}$ correspond to monomials $x^{i} y^{j}$ for $0 \leq i, j<\delta+k$, excluding monomials $x^{i_{0}+i} y^{j_{0}+j}$ for $0 \leq i, j<k$. The columns of lattice $L_{2}$ are obtained from the columns of $L_{2}^{\prime}$ by multiplication with the corresponding $X^{i} Y^{j}$ powers; this gives :

$$
\begin{aligned}
\operatorname{det} L_{2} & =\operatorname{det} L_{2}^{\prime} \cdot \frac{\prod_{0 \leq i, j<\delta+k} X^{i} Y^{j}}{\prod_{0 \leq i, j<k} X^{i_{0}+i} Y^{j_{0}+j}} \\
& =n^{\omega-1} \cdot \frac{(X Y)^{(\delta+k-1) \cdot(\delta+k)^{2} / 2-(k-1) \cdot k^{2} / 2}}{\left(X^{i_{0}} Y^{j_{0}}\right)^{k^{2}}}
\end{aligned}
$$

From inequality (5) we obtain the following condition for Howgrave-Graham's lemma to apply :

$$
2^{\omega \cdot(\omega-1) / 4} \cdot \frac{(X Y)^{(\delta+k-1) \cdot(\delta+k)^{2} / 2-(k-1) \cdot k^{2} / 2}}{\left(X^{i_{0}} Y^{j}\right)^{k^{2}}} \leq \frac{n}{\omega^{\omega / 2}} .
$$

It remains to bound $n=|\operatorname{det} S|$ as a function of the coefficients of $p(x, y)$. Let

$$
W=\max _{i, j}\left|p_{i j}\right| X^{i} Y^{j}
$$

The following lemma shows that for the right choice of $\left(i_{0}, j_{0}\right)$, the determinant of $S$ is bounded in absolute value : 
Lemma 3. Given $(u, v)$ such that $W=\left|p_{u v}\right| X^{u} Y^{v}$, let indices $\left(i_{0}, j_{0}\right)$ that maximize the quantity $8^{(i-u)^{2}+(j-v)^{2}}\left|p_{i j}\right| X^{i} Y^{j}$. Then

$$
\left(\frac{W}{X^{i_{0}} Y^{j_{0}}}\right)^{k^{2}} 2^{-6 k^{2} \delta^{2}-2 k^{2}} \leq|\operatorname{det} S| \leq\left(\frac{W}{X^{i_{0}} Y^{j_{0}}}\right)^{k^{2}} \cdot 2^{k^{2}} .
$$

Proof. The proof is very similar to the proof of Lemma 3 in [7] see Appendix B.

Combining inequalities (9) and (10) with $n=|\operatorname{det} S|$ and $\sqrt{\omega} \leq 2^{\omega / 2}$, we obtain the sufficient condition :

$$
2^{\omega \cdot(\omega-1) / 4} \cdot(X Y)^{(\delta+k-1) \cdot(\delta+k)^{2} / 2-(k-1) \cdot k^{2} / 2} \leq W^{k^{2}} \cdot 2^{-6 k^{2} \delta^{2}-2 k^{2}} \cdot 2^{-\omega^{2} / 2} .
$$

This condition is satisfied if :

$$
X Y<W^{\alpha} \cdot 2^{-9 \delta}
$$

where

$$
\alpha=\frac{2 k^{2}}{\delta \cdot\left(3 k^{2}+k(3 \delta-2)+\delta^{2}-\delta\right)} .
$$

Finally we obtain the sufficient condition :

$$
X Y<W^{2 /(3 \delta)-1 / k} \cdot 2^{-9 \delta} .
$$

The running time is dominated by the time it takes to run LLL on a lattice of dimension $\delta^{2}+2 k \delta$, with entries bounded by $\mathcal{O}\left(W^{k^{2}}\right)$. Namely, the entries of a matrix basis for $L_{2}$ can be reduced modulo $n \cdot X^{i} Y^{j}$ on the columns corresponding to monomial $x^{i} y^{j}$, because of polynomials $r_{i j}(x X, y Y)=n \cdot X^{i} Y^{j} x^{i} y^{j}$. This implies that we can obtain a matrix basis for $L_{2}$ whose entries are bounded by $\mathcal{O}\left(n X^{\delta+k} Y^{\delta+k}\right)$. From inequality (10) we have $n=\mathcal{O}\left(W^{k^{2}}\right)$; using (11) this implies that the matrix entries can be bounded by $\mathcal{O}\left(W^{k^{2}}\right)$. From theorem 1 and taking $k>\delta$, the running time is therefore bounded by :

$$
\mathcal{O}\left(\delta^{6} k^{12} \log ^{3} W\right)
$$

using the LLL algorithm, and $\mathcal{O}\left(\delta^{5} k^{9} \log ^{2} W\right)$ using the improved $L^{2}$ algorithm.

Finally, under the weaker condition

$$
X Y<W^{2 /(3 \delta)},
$$

one can set $k=\lfloor\log W\rfloor$ and do exhaustive search on the high order $\mathcal{O}(\delta)$ unknown bits of $x_{0}$. The running time is then polynomial in $2^{\delta}$ and $\log W$. Moreover, for a fixed $\delta$, the running time is $\mathcal{O}\left(\log ^{15} W\right)$ using the LLL algorithm, and $\mathcal{O}\left(\log ^{11} W\right)$ using the improved $L^{2}$ algorithm. Thus we have shown :

Theorem 2 (Coppersmith). Let $p(x, y)$ be an irreducible polynomial in two variables over $\mathbb{Z}$, of maximum degree $\delta$ in each variable separately. Let $X$ and $Y$ be upper bounds on the desired integer solution $\left(x_{0}, y_{0}\right)$, and let $W=\max _{i, j}$ $\left|p_{i j}\right| X^{i} Y^{j}$. If $X Y<W^{2 /(3 \delta)}$, then in time polynomial in $\left(\log W, 2^{\delta}\right)$, one can find all integer pairs $\left(x_{0}, y_{0}\right)$ such that $p\left(x_{0}, y_{0}\right)=0,\left|x_{0}\right| \leq X$, and $\left|y_{0}\right| \leq Y$. 
As in [7, there can be improved bounds depending on the shape of the polynomial $p(x, y)$ :

Theorem 3 (Coppersmith). With the hypothesis of Theorem [2, except that $p(x, y)$ has total degree $\delta$, the appropriate bound is :

$$
X Y<W^{1 / \delta} .
$$

Proof. See the full version of this paper.

\subsection{Computing a Basis of $L_{2}$}

In the previous section one needs to compute a basis for lattice $L_{2}$, which is then given as input to the LLL algorithm. Such lattice basis can be obtained by triangularization of matrix $M$; a matrix $A$ is upper triangular if $A_{i j}=0$ for $i>j$ (as illustrated in Figure 3). A triangularization algorithm is described in 12 ; for an $m \times n$ matrix of row vectors, its running time is $\mathcal{O}\left(n^{3+\varepsilon} m \log ^{1+\varepsilon} B\right)$ for any $\varepsilon>0$, when the matrix entries are bounded by $B$ in absolute value.

Observe that we don't need to triangularize the full matrix $M$. Namely from our analysis of the previous section, equation (6) can be used to obtain a set of row vectors that generate $L_{2}$; a triangularization algorithm is then applied to derive a lattice basis for $L_{2}$. For this we need to compute matrix $S^{\prime}$ such that $S^{\prime} \cdot S=(\operatorname{det} S) \cdot I$; we note that this is implemented in Shoup's NTL library [22].

Another possibility is to compute the Hermite Normal form (HNF) of $M$. An $m \times n$ matrix $A$ of rank $n$ is in HNF if it is upper triangular and $a_{i i}>0$ for all $1 \leq i \leq n$ and $0 \leq a_{i j}<a_{j j}$ for all $1 \leq j \leq n$ and $1 \leq i<j$. A classical result says that if an $m \times n$ matrix $M$ is of rank $n$ then there exists a $m \times m$ unimodular matrix $U$ such that $U \cdot M$ is in HNF; moreover the HNF is unique. An algorithm for computing the HNF is also described in [12, with the same asymptotic complexity as triangularization. A HNF algorithm is also implemented in Shoup's NTL library 1.

\subsection{Difference with the Algorithm in [9]}

In [9] a similar lattice $L$ is built but with an integer $n$ which is co-prime with the constant coefficient of $p(x, y)$. This implies that the full lattice $L$ must be considered, whose dimension $d_{L}=(\delta+k)^{2}$ grows quadratically with $k$ instead of linearly as in our sub-lattice of dimension $\omega=\delta^{2}+2 k \delta$.

With the full lattice $L$ the LLL fudge factor is then $2^{\left(d_{L}-1\right) / 4}=2^{\mathcal{O}\left(k^{2}\right)}$ instead of $2^{(\omega-1) / 4}=2^{\mathcal{O}(k)}$. This translates in the bound for $X Y$ into the condition $X Y<W^{2 /(3 \delta)-1 / k} \cdot 2^{-\mathcal{O}\left(k^{2}+\delta\right)}$ instead of $X Y<W^{2 /(3 \delta)-1 / k} \cdot 2^{-9 \delta}$. This implies that in [9], in order to reach the bound $X Y<W^{2 /(3 \delta)}$, one must do exhaustive

${ }^{1}$ The LLL algorithms implemented in Shoup's NTL library can in principle receive as input a matrix with $m \geq n$, but for large dimensions we got better results when a lattice basis was provided instead. 
search on the high order $\mathcal{O}\left((\log W) / k+k^{2}\right)$ bits of $X$. The optimum is to take $k:=\mathcal{O}\left(\log ^{1 / 3} W\right)$; this gives a sub-exponential time complexity :

$$
\exp \left(\mathcal{O}\left(\log ^{2 / 3} W\right)\right)
$$

instead of the polynomial-time complexity as in Coppersmith's algorithm and our new algorithm.

\subsection{Extension to More Variables}

Our algorithm can be extended to solve integer polynomial equations with more than two variables, but as for Coppersmith's algorithm, the extension is heuristic only.

Let $p(x, y, z)$ be a polynomial in three variables over the integers, of degree $\delta$ independently in $x, y$ and $z$. Let $\left(x_{0}, y_{0}, z_{0}\right)$ be an integer root of $p(x, y, z)$, with $\left|x_{0}\right| \leq X,\left|y_{0}\right| \leq Y$ and $\left|z_{0}\right| \leq Z$. As for the bivariate case, we can select indices $\left(i_{0}, j_{0}, k_{0}\right)$ that maximize the quantity $X^{i} Y^{j} Z^{k}\left|p_{i j k}\right|$ and consider the matrix $S$ formed by the coefficients of polynomials $s_{a b c}(x, y, z)=x^{a} y^{b} z^{c} \cdot p(x, y, z)$ for $0 \leq a, b, c<m$ for some parameter $m$, but only in the monomials $x^{i_{0}+i} y^{j_{0}+j} z^{k_{0}+k}$ for $0 \leq i, j, k<m$. Then we take $n:=|\operatorname{det} S|$ and define the additional polynomials $r_{i j k}(x, y, z)=x^{i} y^{j} z^{k} n$ for $0 \leq i, j, k<\delta+m$. Then one builds the lattice $L$ formed by all linear combinations of polynomials $s_{a b c}(x X, y Y, z Z)$ and $r_{i j k}(x X, y Y, z Z)$, and consider the sublattice $L_{2}$ obtained by setting to 0 the coefficients of monomials corresponding to matrix $S$. Lattice $L_{2}$ has dimension $\omega=(\delta+m)^{3}-m^{3}$ and using the same analysis as in Section 3. one obtains that $\operatorname{det} L_{2}^{\prime}=n^{\omega-1}$ where $L_{2}^{\prime}$ is the same lattice as $L_{2}$ but without the $X^{i} Y^{j} Z^{k}$ powers.

One then applies LLL to sublattice $L_{2}$; if the ranges $X, Y, Z$ are small enough, we are guaranteed to find a polynomial $h_{1}(x, y, z)$ such that $h_{1}\left(x_{0}, y_{0}, z_{0}\right)=0$ over $\mathbb{Z}$ and $h_{1}(x, y, z)$ is not a multiple of $p(x, y, z)$, but this is not enough. The second vector produced by LLL gives us a second polynomial $h_{2}(x, y, z)$ that can satisfy the same property by bounding its norm as in 3. One can then take the resultant between the three polynomials $p(x, y, z), h_{1}(x, y, z)$ and $h_{2}(x, y, z)$ in order to obtain a polynomial $f(x)$ such that $f\left(x_{0}\right)=0$. But we have no guarantee that the polynomials $h_{1}(x, y, z)$ and $h_{2}(x, y, z)$ will be algebraically independent; this makes the method heuristic only.

\section{Practical Experiments}

As mentioned previously, a direct application of Coppersmith's theorem for the bivariate integer case is to factor $N=p q$ when half of the most significant bits (or least significant bits) of $p$ are known.

Theorem 4 (Coppersmith [7]). Given $N=p q$ and the high-order $1 / 4 \log _{2} N$ bits of $p$, one can recover the factorization of $N$ in time polynomial in $\log N$. 
Namely, given the most significant bits of $p$, one can write :

$$
N=\left(P_{0}+x\right) \cdot\left(Q_{0}+y\right),
$$

where $P_{0}$ and $Q_{0}$ contain the most significant bits of $p$ and $q$. This gives a bivariate integer polynomial equation, for which Theorem 2 can be applied directly. One gets $W=P_{0} \cdot X \simeq N^{1 / 2} \cdot X$ which gives $X Y<W^{2 / 3} \simeq N^{1 / 3} \cdot X^{2 / 3}$. With $X=Y$ this gives $\left|x_{0}\right| \leq X=N^{1 / 4}$.

The result of practical experiments are summarized in Table 1] using Shoup's NTL library 22]. For comparison we have implemented our algorithm and the algorithm in 9]. Table 1 shows that our new algorithm is significantly more efficient; for example, for a 1024-bits modulus with $282=256+26$ bits of $p$ given, our algorithm takes 1 second instead of 13 minutes for the algorithm in [9]; this is due to the fact that LLL is applied on a lattice of smaller dimension.

Table 1. Running times for factoring $N=p q$ given the high-order bits of $p$, using our algorithm and the algorithm in [9], with Shoup's NTL library on a $1.6 \mathrm{GHz}$ PC under Linux

\begin{tabular}{c|c|c||c|c||c|c}
\multicolumn{4}{c||}{ Parameters } & \multicolumn{2}{c||}{ New algorithm } & \multicolumn{2}{c}{ Algorithm in 9 } \\
$N$ & $k$ & bits of $p$ given & Dimension & LLL & Dimension & LLL \\
\hline 512 bits & 4 & 144 bits & 9 & $<1 \mathrm{~s}$ & 25 & $20 \mathrm{~s}$ \\
512 bits & 5 & $141 \mathrm{bits}$ & 11 & $<1 \mathrm{~s}$ & 36 & $2 \mathrm{~min}$ \\
1024 bits & 5 & $282 \mathrm{bits}$ & 11 & $1 \mathrm{~s}$ & 36 & 13 min \\
1024 bits & 12 & $266 \mathrm{bits}$ & 25 & $42 \mathrm{~s}$ & 169 & - \\
\hline
\end{tabular}

The problem of factoring $N=p q$ given the high-order (or low-order) bits of $p$ can also be solved using a simple variant of the one variable modular case, as shown by Howgrave-Graham in [13. Therefore we have also implemented Howgrave-Graham's algorithm to provide a comparison; experimental results are given in Table 2. We obtain that for the particular case of factoring with high-order bits known, our algorithm and Howgrave-Graham's algorithm have roughly the same running time, and work with the same lattice dimension (but the two lattices are different).

Table 2. Running times for factoring $N=p q$ given the high-order bits of $p$, using Howgrave-Graham's algorithm with Shoup's NTL library on a $1.6 \mathrm{GHz}$ PC running under Linux.

\begin{tabular}{c|c|c|c|c}
$N$ & $\mathrm{k}$ & bits of $p$ given & Dimension & LLL \\
\hline $512 \mathrm{bits}$ & 4 & $144 \mathrm{bits}$ & 9 & $<1 \mathrm{~s}$ \\
$512 \mathrm{bits}$ & 5 & $141 \mathrm{bits}$ & 11 & $<1 \mathrm{~s}$ \\
$1024 \mathrm{bits}$ & 5 & $282 \mathrm{bits}$ & 11 & $1 \mathrm{~s}$ \\
$1024 \mathrm{bits}$ & 12 & $266 \mathrm{bits}$ & 25 & $37 \mathrm{~s}$ \\
\hline
\end{tabular}




\section{Conclusion}

We have described a new algorithm for finding small roots of bivariate polynomial equations over the integers, which is simpler than Coppersmith's algorithm but with the same asymptotic complexity. Our simplification is analogous to the simplification brought by Howgrave-Graham for the univariate modular case; it improves on the algorithm in [9] which was not polynomial-time for certain parameters. In practical experiments, our algorithm performs several order of magnitude faster than the algorithm in 9 .

\section{References}

1. Bleichenbacher, D., May, A.: New Attacks on RSA with Small Secret CRTExponents. In: Yung, M., Dodis, Y., Kiayias, A., Malkin, T.G. (eds.) PKC 2006. LNCS, vol. 3958, Springer, Heidelberg (2006)

2. Blomer, J., May, A.: A Tool Kit for Finding Small Roots of Bivariate Polynomials over the Integers. In: Cramer, R.J.F. (ed.) EUROCRYPT 2005. LNCS, vol. 3494, pp. 251-267. Springer, Heidelberg (2005)

3. Boneh, D., Durfee, G.: Crypanalysis of RSA with private key $d$ less than $N^{0.292}$. In: Stern, J. (ed.) EUROCRYPT 1999. LNCS, vol. 1592, Springer, Heidelberg (1999)

4. Boneh, D., Durfee, G., Howgrave-Graham, N.A.: Factoring $n=p^{r} q$ for large $r$. In: Wiener, M.J. (ed.) CRYPTO 1999. LNCS, vol. 1666, Springer, Heidelberg (1999)

5. Coppersmith, D.: Finding a Small Root of a Univariate Modular Equation. In: Maurer, U.M. (ed.) EUROCRYPT 1996. LNCS, vol. 1070, Springer, Heidelberg (1996)

6. Coppersmith, D.: Finding a Small Root of a Bivariate Integer Equation; Factoring with High Bits Known. In: Maurer, U.M. (ed.) EUROCRYPT 1996. LNCS, vol. 1070, Springer, Heidelberg (1996)

7. Coppersmith, D.: Small solutions to polynomial equations, and low exponent vulnerabilities. J. of Cryptology 10(4), 233-260 (1997) Revised version of two articles of Eurocrypt '96

8. Coppersmith, D.: Finding small solutions to small degree polynomials. In: Silverman, J.H. (ed.) CaLC 2001. LNCS, vol. 2146, Springer, Heidelberg (2001)

9. Coron, J.S.: Finding Small Roots of Bivariate Polynomial Equations Revisited. In: Cachin, C., Camenisch, J.L. (eds.) EUROCRYPT 2004. LNCS, vol. 3027, Springer, Heidelberg (2004)

10. Coron, J.S., May, A.: Deterministic Polynomial-Time Equivalence of Computing the RSA Secret Key and Factoring. Journal of Cryptology 20(1) (2007)

11. Ernst, M., Jochemsz, E., May, A., de Weger, B.: Partial Key Exposure Attacks on RSA up to Full Size Exponents. In: Cramer, R.J.F. (ed.) EUROCRYPT 2005. LNCS, vol. 3494, pp. 371-386. Springer, Heidelberg (2005)

12. Hafner, J., McCurley, K.: Asymptotically fast triangularization of matrices over rings. SIAM J. Comput. 20, 1068-1083 (1991)

13. Howgrave-Graham, N.A.: Finding small roots of univariate modular equations revisited. In: Darnell, M. (ed.) Cryptography and Coding. LNCS, vol. 1355, pp. 131-142. Springer, Heidelberg (1997)

14. Howgrave-Graham, N.A.: Approximate integer common divisors. In: Silverman, J.H. (ed.) CaLC 2001. LNCS, vol. 2146, Springer, Heidelberg (2001) 
15. Howgrave-Graham, N.A.: Computational Mathematics Inspired by RSA. PhD thesis, University of Bath (1998)

16. Jochemz, E., May, A.: A Strategy for Finding Roots of Multivariate Polynomials with New Applications in Attacking RSA Variants. In: Lai, X., Chen, K. (eds.) ASIACRYPT 2006. LNCS, vol. 4284, Springer, Heidelberg (2006)

17. Lenstra, A.K., Lenstra Jr., H.W., Lovász, L.: Factoring polynomials with rational coefficients. Mathematische Ann. 261, 513-534 (1982)

18. May, A.: Cryptanalysis of Unbalanced RSA with Small CRT-Exponent. In: Yung, M. (ed.) CRYPTO 2002. LNCS, vol. 2442, pp. 242-256. Springer, Heidelberg (2002)

19. May, A.: Computing the RSA Secret Key is Deterministic Polynomial Time Equivalent to Factoring. In: Franklin, M. (ed.) CRYPTO 2004. LNCS, vol. 3152, pp. 213-219. Springer, Heidelberg (2004)

20. May, A.: Secret Exponent Attacks on RSA-type Schemes with Moduli $N=p^{r} q$. In: Bao, F., Deng, R., Zhou, J. (eds.) PKC 2004. LNCS, vol. 2947, pp. 218-230. Springer, Heidelberg (2004)

21. Nguyen, P.Q., Stehlé, D.: Floating-Point LLL Revisited. In: Cramer, R.J.F. (ed.) EUROCRYPT 2005. LNCS, vol. 3494, Springer, Heidelberg (2005)

22. Shoup, V.: Number Theory C++ Library (NTL) version 5.4. Available at, http://www.shoup.net

23. Shoup, V.: OAEP reconsidered. In: Kilian, J. (ed.) CRYPTO 2001. LNCS, vol. 2139, Springer, Heidelberg (2001)

\section{A Proof of Lemma 1}

Let $R$ be a matrix basis of $L$ and let $U$ be the unimodular matrix such that :

$$
U \cdot M=\left[\begin{array}{c}
R \\
0
\end{array}\right]
$$

(a unimodular matrix $U$ satisfies $\operatorname{det} U= \pm 1$ ). Let $V$ be the unimodular matrix such that $M^{\prime}=M \cdot V$. Then :

$$
U \cdot M \cdot V=U \cdot M^{\prime}=\left[\begin{array}{c}
R^{\prime} \\
0
\end{array}\right],
$$

where $R^{\prime}=R \cdot V$ is a matrix basis for $L^{\prime}$. Then

$$
\operatorname{det} L^{\prime}=\left|\operatorname{det} R^{\prime}\right|=|\operatorname{det}(R \cdot V)|=|\operatorname{det} R| \cdot|\operatorname{det} V|=|\operatorname{det} R|=\operatorname{det} L .
$$

\section{B Proof of Lemma 3}

The proof is very similar to the proof of Lemma 3 in [7. It consists in showing that a matrix related to $S$ is diagonally dominant, which enables to derive a lower bound for its determinant.

Let $W=\max _{i, j}\left|p_{i j}\right| X^{i} Y^{j}$ and let indices $(u, v)$ such that $W=\left|p_{u v}\right| X^{u} Y^{v}$. Let indices $\left(i_{0}, j_{0}\right)$ that maximize the quantity

$$
8^{(i-u)^{2}+(j-v)^{2}}\left|p_{i j}\right| X^{i} Y^{j}
$$


The matrix $S$ is obtained by taking the coefficients of the polynomials $x^{a} y^{b} p(x, y)$ for $0 \leq a, b<k$, taking only the coefficients of monomials $x^{i_{0}+i} y^{j_{0}+j}$ for $0 \leq$ $i, j<k$. We must show :

$$
\left(\frac{W}{X^{i_{0}} Y^{j_{0}}}\right)^{k^{2}} 2^{-6 k^{2} \delta^{2}-2 k^{2}} \leq|\operatorname{det} S| \leq\left(\frac{W}{X^{i_{0}} Y^{j_{0}}}\right)^{k^{2}} 2^{k^{2}}
$$

We let $\mu(i, j)=k i+j$ be an index function; the matrix element $S_{\mu(a, b), \mu(i, j)}$ is the coefficient of $x^{i_{0}+i} y^{j_{0}+j}$ in $x^{a} y^{b} p(x, y)$, namely :

$$
S_{\mu(a, b), \mu(i, j)}=p_{i_{0}+i-a, j_{0}+j-b}
$$

We multiply each $\mu(i, j)$ column of $S$ by

$$
8^{2\left(i_{0}-u\right) i+2\left(j_{0}-v\right) j} X^{i_{0}+i} Y^{j_{0}+j}
$$

and we multiply each $\mu(a, b)$ row by

$$
8^{-2\left(i_{0}-u\right) a-2\left(j_{0}-v\right) b} X^{-a} Y^{-b}
$$

to create a new matrix $S^{\prime}$ whose element is :

$$
S_{\mu(a, b), \mu(i, j)}^{\prime}=p_{i_{0}+i-a, j_{0}+j-b} X^{i_{0}+i-a} Y^{j_{0}+j-b} 8^{2\left(i_{0}-u\right)(i-a)+2\left(j_{0}-v\right)(j-b)}
$$

and we have :

$$
\operatorname{det} S^{\prime}=\operatorname{det} S \cdot\left(X^{i_{0}} Y^{j_{0}}\right)^{k^{2}}
$$

Now we show that $S^{\prime}$ is a diagonally dominant matrix. Let denote $\tilde{p}_{i j}=p_{i j} X^{i} Y^{j}$; the elements of matrix $S^{\prime}$ are :

$$
S_{\mu(a, b), \mu(i, j)}^{\prime}=\tilde{p}_{i_{0}+i-a, j_{0}+j-b} 8^{2\left(i_{0}-u\right)(i-a)+2\left(j_{0}-v\right)(j-b)}
$$

From maximality of $\left(i_{0}, j_{0}\right)$ we have :

$$
\left|\tilde{p}_{i_{0}+i-a, j_{0}+j-b}\right| \cdot 8^{\left(i-a+i_{0}-u\right)^{2}+\left(j-b+j_{0}-v\right)^{2}} \leq\left|\tilde{p}_{i_{0} j_{0}}\right| 8^{\left(i_{0}-u\right)^{2}+\left(j_{0}-v\right)^{2}}
$$

which gives :

$$
\left|\tilde{p}_{i_{0}+i-a, j_{0}+j-b}\right| \cdot 8^{2(i-a)\left(i_{0}-u\right)+2(j-b)\left(j_{0}-v\right)} \leq\left|\tilde{p}_{i_{0} j_{0}}\right| 8^{-(i-a)^{2}-(j-b)^{2}}
$$

and then :

$$
\left|S_{\mu(a, b), \mu(i, j)}^{\prime}\right| \leq\left|\tilde{p}_{i_{0}, j_{0}}\right| 8^{-(i-a)^{2}-(j-b)^{2}}
$$

Each diagonal element $S_{\mu(a, b), \mu(a, b)}^{\prime}$ of matrix $S^{\prime}$ is equal to $\tilde{p}_{i_{0}, j_{0}}$, and using :

$$
\begin{aligned}
\sum_{(i, j) \neq(a, b)} 8^{-(i-a)^{2}-(j-b)^{2}} & \leq \sum_{(i, j) \neq(0,0)} 8^{-i^{2}-j^{2}} \leq-1+\sum_{(i, j)} 8^{-i^{2}-j^{2}} \\
& \leq-1+\left(\sum_{i} 8^{-i^{2}}\right)^{2} \leq \frac{3}{4}
\end{aligned}
$$


we obtain that the sum of the absolute values of the off-diagonal entries in each $\mu(a, b)$ row is at most $\frac{3}{4}\left|\tilde{p}_{i_{0}, j_{0}}\right|$. Therefore matrix $S^{\prime}$ is diagonally dominant and each eigenvalue $\lambda$ must verify :

$$
\frac{1}{4}\left|\tilde{p}_{i_{0}, j_{0}}\right| \leq|\lambda| \leq \frac{7}{4}\left|\tilde{p}_{i_{0}, j_{0}}\right|
$$

which gives :

$$
\left|\tilde{p}_{i_{0}, j_{0}}\right| k^{2} 2^{-2 k^{2}} \leq\left|\operatorname{det} S^{\prime}\right| \leq\left|\tilde{p}_{i_{0}, j_{0}}\right|^{k^{2}} 2^{k^{2}}
$$

From the optimality of $\left(i_{0}, j_{0}\right)$, we have :

$$
8^{\left(i_{0}-u\right)^{2}+\left(j_{0}-v\right)^{2}}\left|\tilde{p}_{i_{0}, j_{0}}\right| \geq 8^{0+0}\left|\tilde{p}_{u, v}\right|=W
$$

which gives :

$$
8^{-2 \delta^{2}} W \leq\left|\tilde{p}_{i_{0}, j_{0}}\right| \leq W
$$

Combining with (14) we obtain :

$$
W^{k^{2}} 2^{-6 k^{2} \delta^{2}-2 k^{2}} \leq\left|\operatorname{det} S^{\prime}\right| \leq W^{k^{2}} \cdot 2^{k^{2}}
$$

and using (13) we obtain (12). 